\title{
О ГЛАДКОСТИ ОБОБЩЕННЫХ РЕШЕНИЙ ПЕРВОЙ КРАЕВОЙ ЗАДАЧИ ДЛЯ \\ ФУНКЦИОНАЛЬНО-ДИФФЕРЕНЦИАЛЬНОГО \\ УРАВНЕНИЯ С ОРТОТРОПНЫМИ СЖАТИЯМИ ВНУТРИ ОБЛАСТИ
}

@ А.Л. Тасевич

tasevich-al@rudn.ru

\section{УДК 517.954}

DOI: $10.33184 /$ mnkuomsh1t-2021-10-06.84.

Рассмотрены результаты по исследованию гладкости обобщенных решений задачи Дирихле для сильно эллиптического функциональнодифференциального уравнения с ортотропными сжатиями в плоской области. Сильная эллиптичность определяется выполнением неравенства типа Гординга.

Ключевые слова: функционально-дифференциальное уравнение, гладкость обобщенных решений, оператор сжатия, нелокальные задачи, сильно эллиптическое уравнение.

On smoothness of generalized solutions of the first boundary value problem for functional differential equation with orthotropic contractions inside the domain

The results on study the smoothness of generalized solutions of the Dirichlet problem for strongly elliptic functional differential equation with orthotropic contractions in the plane domain are considered. The strongly ellipticity is defined by holding the Garding-type inequality.

Keywords: functional differential equations, smoothness of generalized solutions, contraction operator, nonlocal problems, strongly elliptic equations.

Рассмотрим первую краевую задачу для функционально-дифференциального уравнения

$$
\begin{gathered}
A_{R} u\left(x_{1}, x_{2}\right) \equiv-\sum_{i, j=1}^{2}\left(R_{i j} u_{x_{i}}\left(x_{1}, x_{2}\right)\right)_{x_{j}}=f\left(x_{1}, x_{2}\right), \quad\left(x_{1}, x_{2}\right) \in \Omega, \\
\left.u\right|_{\partial \Omega}=0 .
\end{gathered}
$$

Работа выполнена при финансовой поддержке Минобрнауки РФ в рамках государственного задания (номер темы FSSF-2020-0018)

Тасевич Алла Львовна, к.ф.-м.н., ассистент, Российский университет дружбы народов (Москва, Россия); Alla Tasevich (RUDN University, Moscow, Russia) 
в области $\Omega \subset \mathbb{R}^{2}$. Здесь оператор $R_{i j}$ определяется по формуле

$$
R_{i j} v\left(x_{1}, x_{2}\right)=a_{i j 0} v\left(x_{1}, x_{2}\right)+a_{i j 1} v\left(q_{1}^{-1} x_{1}, q_{2}^{-1} x_{2}\right)+a_{i j,-1} v\left(q_{1} x_{1}, q_{2} x_{2}\right) .
$$

В рассматриваемой задаче числа $q_{1}, q_{2}>0$, коэффициенты уравнения $a_{i j 0}, a_{i j, \pm 1} \in \mathbb{C}(i, j=1,2)$, а функция $f \in L_{2}(\Omega)$ является комплекснозначной. Также, мы предполагаем, что если для некоторой точки $\left(\tilde{x}_{1}, \tilde{x}_{2}\right) \in \Omega$ преобразованная точка $\left(\tilde{x}_{1} / q_{1}^{ \pm 1}, \tilde{x}_{2} / q_{2}^{ \pm 1}\right)$ оказывается вне области, то $v\left(\tilde{x}_{1} / q_{1}^{ \pm 1}, \tilde{x}_{2} / q_{2}^{ \pm 1}\right)=0$.

Сформулируем теперь определение сильной эллиптичности следующим образом.

Определение 1. Уравнение (1) будем называть сильно эллиптическим уравнением, а соответствующий оператор $A_{R}$ - сильно эллиптическим оператором, если существуют такие постоянные $c_{1}>0, c_{2} \geq 0$, что для любой функции $u \in C_{0}^{\infty}(\Omega)$ выполняется неравенство типа Гординга

$$
\operatorname{Re}\left(A_{R} u, u\right)_{L_{2}(\Omega)} \geqslant c_{1}\|u\|_{H^{1}(\Omega)}^{2}-c_{2}\|u\|_{L_{2}(\Omega)}^{2} .
$$

Задачу о нахождении алгебраических условий на коэффициенты уравнения (1), при которых оператор $A_{R}$ будет сильно эллиптическим, называют проблемой коэрцитивности. Исследование достаточных и необходимых условий выполнения неравенства Гординга уравнения было проведено в [1].

Определение 2. Функция $u \in \stackrel{\circ}{H}^{1}(\Omega)$ называется обобщенным решением задачи 1 , если интегральное тождество

$$
a_{R}[u, v]:=\sum_{i, j=1}^{2}\left(R_{i j} u_{x_{i}}, v_{x_{j}}\right)_{L_{2}(\Omega)}=(f, v)_{L_{2}(\Omega)}
$$

выполнено для любой функции $v \in \stackrel{\circ}{H}^{1}(\Omega)$.

Будут справедливы следующие теоремы о гладкости обобщенных решений.

Теорема 1. Пусть уравнение (1) является сильно эллиптическим в замыкании $\bar{\Omega}$. Предположим, что функция и является обобщенным решением краевой задачи (1), (2), а функция $f \in L_{2}(\Omega) \cap H_{l o c}^{k}\left(\Omega_{s l}\right) \quad(s \in$ $\mathbb{N}, l=\overline{1, l(s)})$. Тогда $u \in H_{l o c}^{k+2}\left(\Omega_{s l}\right)$ для всех $s, l$.

Теорема 2. Пусть уравнение (1) является сильно эллиптическим в замыкании $\bar{\Omega}$, а также $q_{1}>1,1 / q_{2}>1$. Предположим, что функиия и является обобщенным решением краевой задачи (1), (2), а функиия $f \in L_{2}(\Omega) \cap H^{k}\left(\Omega_{s l}\right)(s \in \mathbb{N}, l=\overline{1, l(s)})$. Тогда $u \in H^{k+2}\left(\Omega_{s l} \backslash \overline{\mathcal{K}^{\varepsilon}}\right)$ для всех $\varepsilon>0(s \in \mathbb{N}, l=\overline{1, l(s)})$, где $\mathcal{K}^{\varepsilon}=\left\{x \in \mathbb{R}^{2}: \rho(x, \mathcal{K})<\varepsilon\right\}$. 
Схему построения подобластей $\Omega_{s l}$ и множества $\mathcal{K}$, а также доказательства теорем можно найти в [2], где в качестве области рассмотрен круг с центром в начале координат.

Дополнительную информацию о разрешимости краевой задачи (1), (2) в пространствах Соболева и весовьх пространствах можно найти в $[3,4]$.

\section{Литература}

1. Россовский Л.Е., Тасевич А.Л. Первая краевая задача для сильно эллиптического функцинально-дифференциального уравнения с ортотропными сжатиями // Матем. сборник, 97:5 (2015), 733-748.

2. Тасевич А.Л. Гладкость обобщенных решений задачи Дирихле для сильно эллиптических функционально-дифференциальных уравнений с ортотропными сжатиями // Современная математика. Фундаментальные направления, 58 (2015), 153-165.

3. Россовский Л.Е., Тасевич А.Л. Об однозначной разрешимости функциональнодифференщиальных уравнений с ортотропными сжатиями в весовых пространствах // Дифференц. уравнения, 53:12 (2017), 1679-1692.

4. Tasevich A.L. Analysis of Functional-Differential Equation with Orthotropic Contractions // Math. Model. Nat. Phenom., 12:6 (2017), 240-248. 